\title{
Separation of Steryl Glucoside by Using Various Guard Columns with 100-А Phenogel Column
}

\author{
Apinya Cheewaphan ${ }^{1}$, Kanit Krisnangkura ${ }^{2}$, Kornkanok Aryusuk ${ }^{3}$, \\ Narumon Jeyashoke ${ }^{4}$, and Supathra Lilitchan ${ }^{5}$ \\ ${ }^{1,2,3,4}$ Division of Biochemical Technology, School of Bioresources and Technology, \\ King Mongkut's University of Technology Thonburi (Bangkhuntien), Bangkok, Thailand. \\ ${ }^{1}$ Department of Basic Medical Science, Faculty of Medicine Vajira Hospital, \\ Navamindradhiraj University, Bangkok, Thailand. \\ ${ }^{5}$ Department of Nutrition, Faculty of Public Health, Mahidol University, Rachathewi, Bangkok 10400, Thailand.
}

\begin{abstract}
Steryl glucoside (SG) are the minor component in plant material and biodiesel. SG are found in many forms, especially linked to a glucoside moiety (SG). The presence of SG may relate to fuel filter plugging problem. SG would be slowly precipitated in the fuel tank. Complete separation may take days or weeks and may accumulate and plug the fuel filter. Accordingly, High-performance size-exclusion chromatography (HPSEC) is the method to analyze SG. Phenogel column was very interesting that all the lipids were separated into their classes, which facilitated the SG separation. This separation on the swelling-controlled styrene-divinylbenzene (ST-DVB) copolymer (phenogel) column. In this work, SG and lipid standards (triacylglycerols; TG, diacylglycerols; DG), fatty acid methyl esters; FAME), free fatty acids; FFA), and monoacylglycerols; MG were investigated by using HPSEC with phenogel column connected with various guard columns. Use guard column with the identical packing materials as in the analytical and preparative columns. Even the guard column has slightly different packing materials that might cause improved separation in mixture compounds. The results demonstrated that the phenogel column connected with C18 $(20 \mathrm{~mm})$ guard column by using toluene/THF (100:20, v/v) was suitable for SG separation and shorter analysis time than the others. Thus, this work would demonstrate the $\mathrm{C} 18$ guard column should be improved the SG separation from the others and biodiesel products.
\end{abstract}

Keywords: Biodiesel, Guard column, High-performance size-exclusion chromatography, ST-DVB, Steryl glucoside.

\section{Introduction}

Phytosterols are bioactive compounds that occur in trace levels in various plants. The phytosterols have been reported to include over 250 different sterols. The most important natural sources of plant sterols are animal fats and vegetable oils. They were also found in a 
range of seeds, legumes, vegetables, and unrefined vegetable oils (Marangoni \& Poli, 2010). They made up the largest proportion of the unsaponifiable fraction of lipids (Whitaker \& Hicks, 2002).

Sterols are some of the minor components in fats and oils and are found in many forms, such as free sterols, sterol esters, or linked to a glucose moiety (SG). Among the several common sterols, SG have been found to be a major component of biodiesel precipitates (Wang et al., 2010). Nowadays, biodiesel is an alternative renewable fuel. It is biodegradable, environmentally friendly, and also can be manufactured from fat and oil feedstocks. Biodiesel was generally produced by transesterification of triglyceride (TG) with a short-chain alcohol using an alkaline catalyst. Typically, this fuel contained minor amounts of acylglycerols (unreacted TG, DG, MG), FFA, and unsaponifiable matter (Kittirattanapiboon \& Krisnangkura, 2008).

When biodiesel had high concentration of unsaponifiable matter, SG (Fig. 1) tended to form white precipitate, even the amount of SG in biodiesel were less than $100 \mathrm{mg} / \mathrm{kg}$ (Lacoste et al., 2009). As a result of the hydroxyl groups in SG that raised its polarity and rendering its very low solubility in biodiesel or biodiesel- petrodiessel blend (Van Hoed et al., 2008). SG have been ascribed as a cause for filter plugging in engine that used biodiesel as fuel extender (NaRanong et al., 2015; Plata et al., 2020).

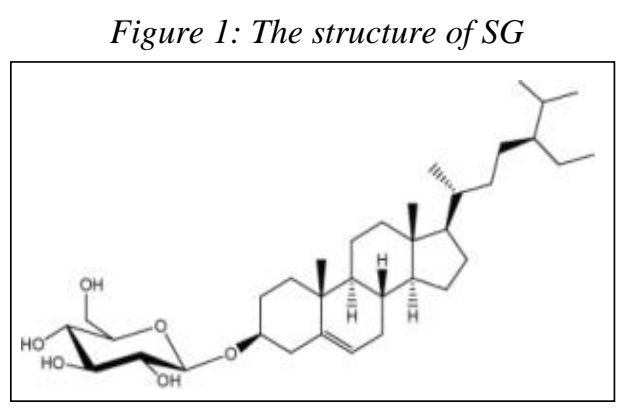

Various analytical methods have been developed for the quantification of SG. For example, a pre-fractionation of the lipid sample to eliminate the main components, triglycerides or FAME, and a chromatographic technique with or without derivatization. Some analytical methods have been reported for direct quantification of steryl esters without removal of TG. Steryl esters and free sterols, were separated from TG by TLC or normal-phase LC and quantified by GC or HPLC. This direct analysis of the SG always led to an incomplete separation of the different SG (Verleyen et al., 2002).

Verleyen, et al. showed that the phytosterols (corn and rapeseed oil), which mainly occurred as steryl esters (56-60\%) were separated by silica gel column chromatography and quantified by GC (Verleyen et al., 2002). Column chromatography could separate lipids into different classes (Sanjay S. et al., 2020). Arzamendi et al. monitored the biodiesel products from transesterification by using high performance size exclusion chromatography (HPSEC) (Arzamendi et al., 2006). However, three columns of different pore sizes serially connected were required for complete separation. HPSEC is a technique for separation based on solutes molecular size. It is appropriate for separation lipids into different classes or molecular size, 
which normally related to their molecular weight (MW) of similar shape. Phenogel column is designed for use as size exclusion chromatographic column packed with styrenedivinylbenzene (ST-DVB) copolymer. As pointed out above that Arzamendi et al. made use of HPSEC to separate TG, DG, MG, FAME, free glycerol, and methanol in the biodiesel reactor (Arzamendi, et al., 2006). By mobile phase engineering, Kittirattanapiboon and Krisnangkura showed that acylglycerols, FAME, and FFA in the biodiesel reactor could be baseline separated by using a single phenogel columns (100 ̊) (Kittirattanapiboon \& Krisnangkura, 2008). Also, Chumsantea et al. demonstrated that vitamin E homologs was baseline separated on a swellingcontrolled phenogel column $(100-\AA)($ Chumsantea et al., 2017). Essentially, the gel matrix pore size, mobile phase, type and dimension of the guard column were greatly affecting the effectiveness of the separation. These effects were reported by Aryusuk et al. (2011) on the separation of TG and wax and by (Krisnangkura \& Simamaharnnop, 1992)

In theory, the same phase chemistry for guard and column, the efficiency should simply improve. Even with identical phase, it is not impossible that the guard and column have slightly different selectivities which might sometimes cause improved separation in some particular case. For example, the use of a guard column to eliminate separation complications. As the result, Aryusuk et al. were determined the content of wax in a single run on the modified 100$\AA$ phenogel column connected with C18 guard column to separate the lipids in crude rice bran wax (Aryusuk et al., 2011). On the other hands, phenogel column is protected with a column guard packed with silica, which has a beneficial effect on the separation of diglyceride and palm oil (Krisnangkura \& Simamaharnnop, 1992).

The objective of this work was to develop an effective method for determination of SG in biodiesel. The effect of guard columns on SG separation by using HPSEC with Phenogel column was thoroughly investigated.

\section{Experiment}

\subsection{Chemicals}

Tripalmitin (TG), 1,3-dipalmitin (DG), FAME, Oleic acid (FFA), and 1-stearoyl-racglycerol (MG) standards were purchased from Sigma- Aldrich (Louis, MO, USA). SG standard was purchased from Matreya LLC (PA, USA). THF and toluene were obtained from RCI Lab Scan Co., Ltd. (Bangkok, Thailand).

\subsection{High Performance Size- Exclusion Chromatography (HPSEC)}

The HPLC system consisted of a Waters model 510 pump system (Waters Associate, Milford, MA, USA.) equipped with a Rheodyne model 7125 six-port injector (Cotati, CA, USA) coupled to an evaporative light scattering detector (ELSD) model 80 from SEDEX (Sedere, Alfortville, France).

All standards were prepared in THF at concentration of $1 \mathrm{mg} / \mathrm{mL}$ and analyzed on a $100-\AA$ Phenogel column (300 x $7.8 \mathrm{~mm}$. ID., $5 \mu \mathrm{m})$ (Phenomenex, Torrance, CA, USA) connected with different guard columns (phenogel $(50 \mathrm{~mm}), \mathrm{C} 18(20 \mathrm{~mm})$, silica 5, 10, and $20 \mathrm{~mm})$. The injector and column were set at $65^{\circ} \mathrm{C}$. The ELSD drift tube was set at $30^{\circ} \mathrm{C}$ and the nitrogen flow through the nebulizer was set at two bars. Peaks were identified by comparison with 
$3^{\text {rd }}$ International Conference on Advanced Research in Applied SCIENCE \& ENGINEERING

reference standards. The mobile phase ratios consisted of toluene/THF (100:20, v/v) and toluene/THF (80:20, v/v). The flow rate of each mobile phase was $1 \mathrm{~mL} / \mathrm{min}$. 


\subsection{Results and Discussion}

\subsubsection{Effect of toluene size-exclusion chromatography}

Figure 2: The chromatograms of SG separation on a 100-A phenogel column.

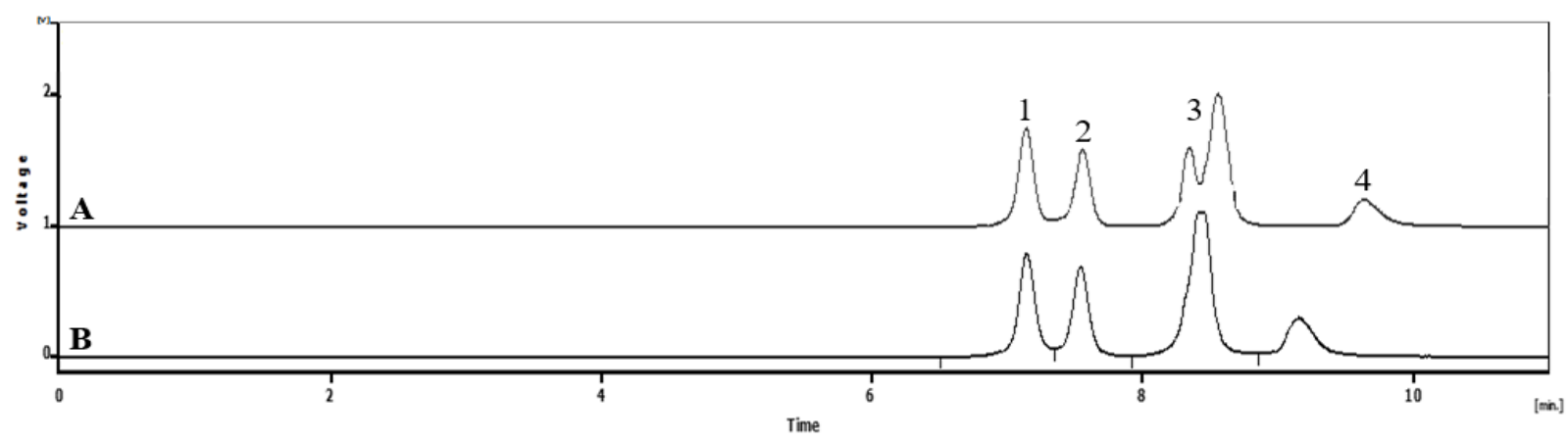

Noted: A 100-A phenogel column connected with C18 $(20 \mathrm{~mm})$ guard column with the various mobile phases; (A) toluene/THF $(100 / 20, v / v)$, and (B) toluene/THF (80/20, v/v), respectively. All standards; (1) TG, (2) DG, (3) $F A M E$, and FFA with $M G$, and (4) $S G$, respectively.

Toluene and THF was the mobile phase for separation of SG and other lipids. Fig. 2A, toluene-THF were 100:/20, v/v) and Fig. 2B, toluene-THF were 80:20, v/v. Both toluene and THF were good swelling solvents for the cross-link vinyl-divinyl benzene copolymer beads, the gel matrix of Phenogel. THF was added to control the polarity of the mobile phase. Thus, FAMEs, FFA, and MG, which have approximately the same molcular weigth eluted according to their binding capacity to the gel matrix. Fig. 2B shows that these solutes are coeluted when toluene-THF 80:20 are the mobile phase. Partial separation of FAME from FFA and MG is oberved in Fig 2A when the toluene-THF is increase to 100:20 v/v and polarity of the mobile phase is decreased. FAME, which has no active hydrogen is not affected and eluted at the same time as that in Fig. 2B, while FFA and MG have an acid proton and two active hydroxy hydrogens, respectively are eluted slower or have longer retention time. SG, which has four active hydrogens shows a larger shift in retention time. 
$3^{\text {rd }}$ International Conference on Advanced Research in

Applied SCIENCE \& ENGINEERING

02-04 JULY, 2021

Oxford, United Kingdom

\subsubsection{Effect of guard columns with a 100-Å phenogel column on separation of SG}

Figure 3: Separation of SG and others lipid with various guard columns on a 100-A phenogel column eluted. Toluene-THF (100:20, v/v

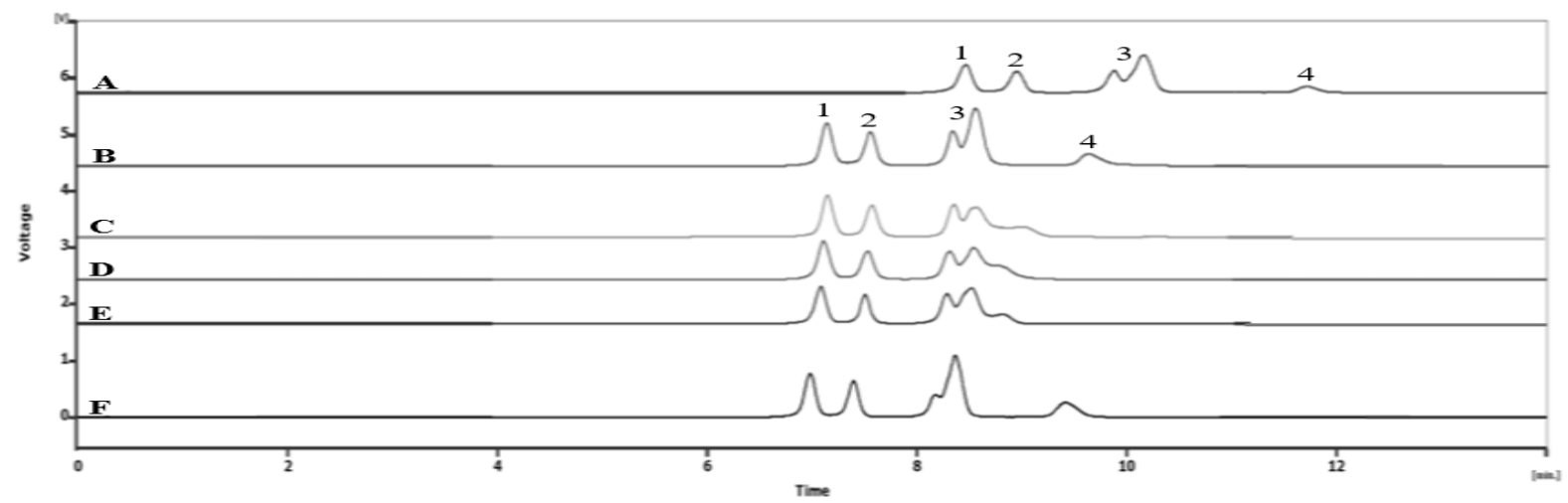

Noted: Condition: A 100-A phenogel column connected with the various guard columns; (A) phenogel guard column (50 mm), (B) C18 guard column $(10 \mathrm{~mm}),(C)$ silica guard column $(20 \mathrm{~mm}),(D)$ silica guard column $(10$ mm), (E) silica guard column (5 mm), and (F) no guard column). Peaks: (1), TG; (2), DG; (3), FAME and FFA with $M G$, and (4) $S G$.

Guard column is normally used to protect the analytical or preparative column from particulates deposit on the column head. Consequently, it prolongs its column life. Both the scientific literature and the commercial column suppliers focus on the protection of the column rather than the modification to increase the efficiency of the column (Shihabi, 1988). Ideally, the guard column packed with the same packing material as the analytical column is chosen. In this study, different packing material were employed to enhanced separation efficiency. Phenogel (50 mm), C18 (20 mm), silica 5, 10, and $20 \mathrm{~mm}$ guard columns were investigated.

Result in Fig. 3A shows that the SG can be separated from other lipids on the phenogel column using the phenogel guard column but FAMEs can be partially separated from FFA, and MG, which were co-eluted. Similarly, C18 guard showed very similar separation to phenogel guard column, but all the lipids were eluted at much shorter retention times. On the other hand, silica gel guard columns of 5, 10, and $20 \mathrm{~mm}$ could separate FAMEs from FFA and MG but they were not baseline separated. SG was strongly adsorbed by silica gel and did not eluted out of the column (Fig 3, C-E). Column without a column guard (Fig. 3F) shows similar separation to that of the Phenogel guard column but all the solutes eluted at a slightly shorter retention times. This is not unexpected, because the total column length was reduced.

\section{Conclusion}

The 100-A phenogel column connected with C18 (20 mm) guard column using toluene-THF $(100: 20, \mathrm{v} / \mathrm{v})$ as the mobile phase is best for separation of SG from other biodiesel reaction products. The proposed method provided a rapid determination of SG and other minor components in biodiesel reactor and storage tank. Its saved both analysis time and mobile solvents. 


\section{Acknowledgment}

The authors are gratefully acknowledged financial support from Faculty of Medicine Vajira Hospital, Navamindradhiraj University and King Mongkut's University of Technology Thonburi for funding the KMUTT Research Center of Excellence Project to Lipid Technology Research Group.

\section{References}

Aryusuk, K., Chumsantea, S., Sombatsuwan, P., Lilitchan, S., \& Krisnangkura, K. (2011). Separation and Determination of Wax Content Using 100- $\AA$ Phenogel Column. Journal of the American Oil Chemists' Society, 88(10), 1497-1501. https://doi.org/https://doi.org/10.1007/s11746-011-1816-X

Arzamendi, G., Arguiñarena, E., Campo, I., \& Gandía, L. M. (2006). Monitoring of biodiesel production: Simultaneous analysis of the transesterification products using sizeexclusion chromatography. Chemical Engineering Journal, 122(1), 31-40. https://doi.org/https://doi.org/10.1016/j.cej.2006.05.009

Chumsantea, S., Aryusuk, K., Lilitchan, S., Jeyashoke, N., \& Krisnangkura, K. (2017). Separation of Vitamin E on a 100-Å Phenogel Column. J Chromatogr Sci, 55(2), 149153. https://doi.org/10.1093/chromsci/bmw158

Kittirattanapiboon, K., \& Krisnangkura, K. (2008). Separation of acylglycerols, FAME and FFA in biodiesel by size exclusion chromatography. European Journal of Lipid Science and Technology, 110(5),

https://doi.org/https://doi.org/10.1002/ejlt.200700164

Krisnangkura, K., \& Simamaharnnop, R. (1992). Continuous transmethylation of palm oil in an organic solvent. Journal of the American Oil Chemists' Society, 69(2), 166-169.

Lacoste, F., Dejean, F., Griffon, H., \& Rouquette, C. (2009). Quantification of free and esterified steryl glucosides in vegetable oils and biodiesel. European Journal of Lipid $\begin{array}{llll}\text { Science } \quad \text { and } & \text { Technology, }\end{array}$ https://doi.org/https://doi.org/10.1002/ejlt.200800297

Marangoni, F., \& Poli, A. (2010). Phytosterols and cardiovascular health. Pharmacol Res, 61(3), 193-199. https://doi.org/10.1016/j.phrs.2010.01.001

Na-Ranong, D., Laungthaleongpong, P., \& Khambung, S. (2015). Removal of steryl glucosides in palm oil based biodiesel using magnesium silicate and bleaching earth. Fuel, 143, 229-235. https://doi.org/https://doi.org/10.1016/j.fuel.2014.11.049

Plata, V., Rojas, Ó., \& Gauthier-Maradei, P. (2020). Improvement of palm oil biodiesel filterability by treatment with reactivated spent bleaching earths. Fuel, 260, 116198. https://doi.org/https://doi.org/10.1016/j.fuel.2019.116198

Sanjay S., Manish S., Rupesh Kumar G, \& Goyal, S. (2020). Columns in Pharmaceuticals: For Primers. Indian Journal of Pharmaceutical Education and Research, $54(3$ (Suppl)), 423-432. 
Van Hoed, V., Zyaykina, N., De Greyt, W., Maes, J., Verhé, R., \& Demeestere, K. (2008). Identification and Occurrence of Steryl Glucosides in Palm and Soy Biodiesel. Journal of the American Oil Chemists' Society, 85(8), 701. https://doi.org/10.1007/s11746-008$\underline{1263-5}$

Verleyen, T., Forcades, M., Verhe, R., Dewettinck, K., Huyghebaert, A., \& De Greyt, W. (2002). Analysis of free and esterified sterols in vegetable oils. Journal of the American Oil Chemists' Society, 79(2), 117-122. https://doi.org/10.1007/s11746-002-0444-3

Wang, H., Tang, H., Salley, S., \& Ng, K. Y. S. (2010). Analysis of Sterol Glycosides in Biodiesel and Biodiesel Precipitates. Journal of the American Oil Chemists' Society, 87(2), 215-221. https://doi.org/https://doi.org/10.1007/s11746-009-1489-X

Whitaker, B., \& Hicks, K. (2002). Phytosterols, phytostanols, and their conjugates in foods: Structural diversity, quantitative analysis, and health-promoting uses. Progress in lipid research, 41, 457-500. https://doi.org/10.1016/S0163-7827(02)00006-1 